\title{
Tropheryma whipplei endocarditis: The value of valve polymerase chain reaction in cardiac surgery
}

\author{
David Rose, MD, ${ }^{\mathrm{a}}$ Achyut Guleri, MD, ${ }^{\mathrm{b}}$ and Augustine Tang, FRCS (CTh), ${ }^{\mathrm{a}, \mathrm{c}}$ Blackpool and Bailrigg, \\ Lancashire, United Kingdom
}

Video clip is available online.

Tropheryma whipplei is the etiologic agent of Whipple's disease, a chronic infection characterized by diarrhea, weight loss, intra-abdominal lymphadenopathy, and arthropathy. The disease is extremely rare, with an estimated incidence of $<1 / 1,000,000$, and cardiac involvement has been reported in $17 \%$ to $55 \%$ of patients with classic Whipple's disease, with pericarditis the most frequent. We report a case of a patient with a very rare case of $T$ whipplei pancarditis in whom the cardiac involvement characterized the initial presentation without any intestinal symptoms. Isolate identification was confirmed by DNA nucleotide sequencing of explanted valve tissue in response to a high index of suspicion of an infectious process arising from intraoperative findings, despite negative results for routine cultures.

\section{CASE REPORT}

A previously fit and active 71-year-old man presented with non-ST-segment myocardial infarction and respiratory failure requiring ventilation in the intensive care unit. Transthoracic echocardiography revealed severe aortic regurgitation and severe mitral regurgitation. The noncoronary aortic cusp and adjacent annulus appeared thickened, with restricted movement (Figure 1, A, and Video 1). The posterior mitral valve leaflet was thickened at the base, with additional nodular thickening of the anterior leaflet (Figure 1, B). The coronary angiogram showed severe triple vessel disease. His left ventricular ejection fraction was $45 \%$. Intraoperatively, the pericardial space was filled with unusually dense adhesions, with multiple calcified nodules scattered across the epicardium (Figure 1,C). After

\footnotetext{
From the Lancashire Cardiac Centre, ${ }^{a}$ Blackpool, Lancashire, United Kingdom; Department of Clinical Microbiology, ${ }^{\mathrm{b}}$ Blackpool Victoria Hospital, Blackpool, Lancashire, United Kingdom; and Lancaster University School of Health and Medicine, ${ }^{\mathrm{c}}$ Bailrigg, Lancashire, United Kingdom.

Disclosures: Achyut Guleri reports lecture fees from Pfizer, BD Diagnostics, and Novartis.

All other authors have nothing to disclose with regard to commercial support.

Received for publication April 4, 2014; revisions received May 11, 2014; accepted for publication May 14, 2014; available ahead of print June 18, 2014.

Address for reprints: David Rose, MD, Lancashire Cardiac Centre, Whinney Heys

Rd, Blackpool, Lancashire FY3 8NR, UK (E-mail: davidrose@libero.it).

J Thorac Cardiovasc Surg 2014;148:e195-7

$0022-5223 / \$ 36.00$

Copyright (C) 2014 by The American Association for Thoracic Surgery

http://dx.doi.org/10.1016/j.jtcvs.2014.05.045
}

cardioplegic arrest and triple bypass grafting, the aortic root was inspected. The inspection showed the noncoronary cusp had been entirely destroyed adjacent to the abscess (Figure 1,D). The annular tissue was grossly thickened and fibrosed. After deroofing the abscess, grayish pus was evacuated. After debridement, the cavity walls were plicated to eliminate the space. Gross thickening was found in both mitral leaflets and the subchordal apparatus. Two separate chronic abscess cavities were present along the posterior annulus (Figure 1,E). After debridement and annular reconstruction using a tailored pericardial patch, a bioprosthesis was implanted with papillary muscle resuspension. Routine cultures of the valve specimens gave negative results. Histopathologic examination showed focal myxoid degeneration with fragments of partially hyalinized fibrous connective tissue and extensive amorphous dystrophic calcification, accompanied by chronic inflammation, including histiocytes, macrophages, neutrophils, and plasma cells (Figure 1, F).

Identification of $T$ whipplei was confirmed using a DNA amplification technique (polymerase chain reaction) plus sequencing. This enabled optimization of the postoperative treatment with oral co-trimoxazole $960 \mathrm{mg}$ (trimethoprim/sulfamethoxazole) after surgery for 12 months. The postoperative findings were uneventful at 8 months of follow-up, with no echocardiographic or clinical evidence of recurrent infection.

\section{DISCUSSION}

Whipple's disease is a rare condition, first described by George Hoyt Whipple in 1907, caused by T whipplei. This organism belongs to the gram-positive actinomycetes, and some studies have shown a statistically significant greater prevalence in those in the farming profession compared with the incidence in those in other occupations. It has usually presented with gastrointestinal symptoms, affecting the cardiovascular system to a lesser extent. ${ }^{1}$ The disease is extremely rare, with an estimated incidence of $<1 /$ $1,000,000$. It is strongly prevalent in white men, with a male to female ratio of $8: 1$ and a mean age of onset around 50 years. ${ }^{2}$ Cardiac involvement has been reported in $17 \%$ to $55 \%$ of patients with classic Whipple's disease, with pericarditis the most common. ${ }^{3}$ It is very rare that cardiac involvement is the primary and sole presentation in the absence of intestinal symptoms. T whipplei infective endocarditis is difficult to diagnose because blood cultures will often fail to reveal the organism, resulting in $\leq 31 \%$ of 

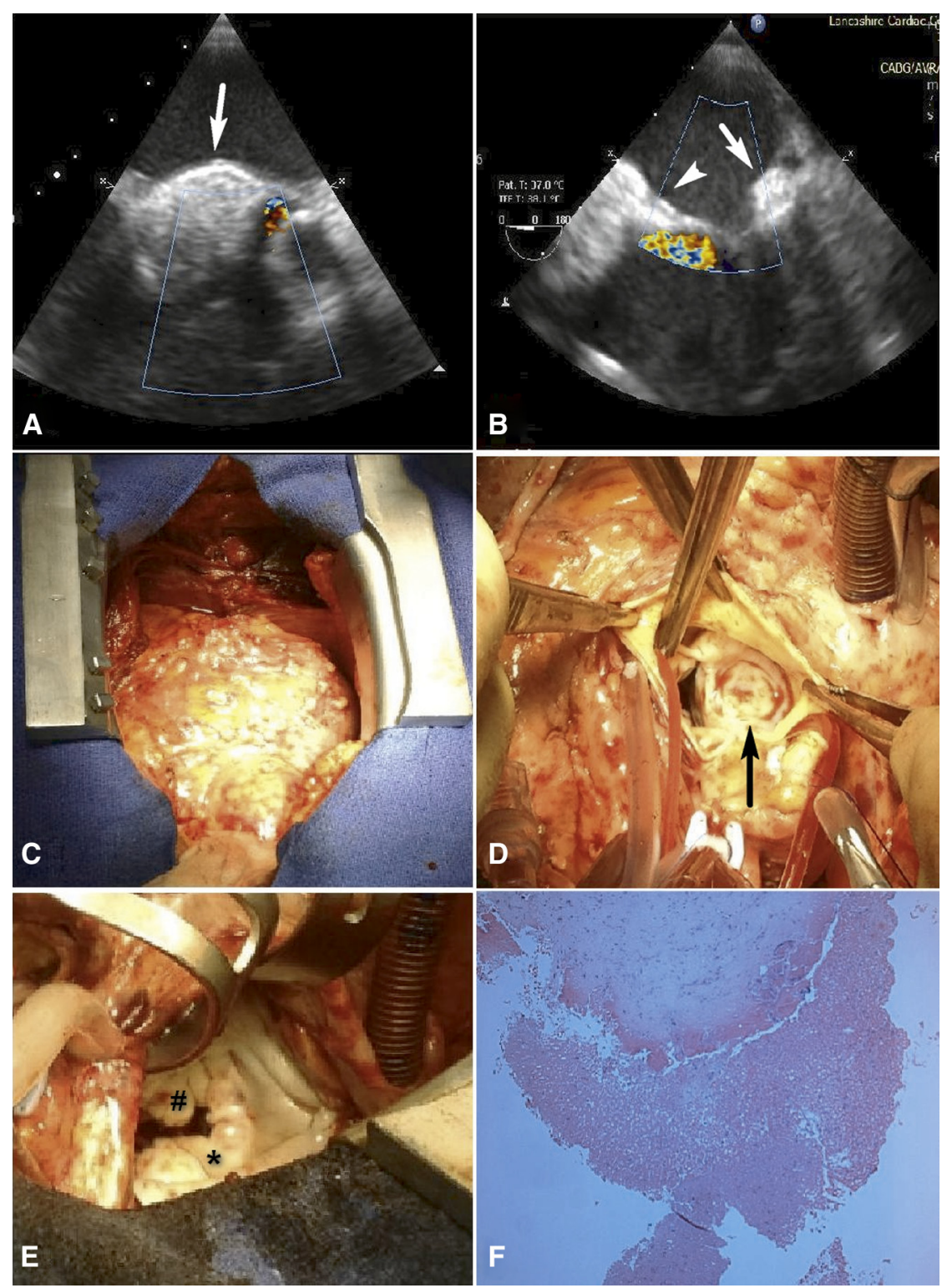

FIGURE 1. A, Detail of the noncoronary aortic cusp appearing thickened (white arrow). B, Mitral valve with the posterior leaflet thickened at the base (white arrow) and additional nodular thickening of the anterior leaflet (white arrow tip). C, Intraoperative aspect of the pericardium presenting with multiple calcified nodules scattered across the epicardium. D, Intraoperative aspect of the aortic valve, with the noncoronary cusp adjacent to the abscess entirely destroyed (black arrow). E, Intraoperative aspect of the mitral valve presenting with gross thickening in both mitral leaflets (pound sign, anterior leaflet; asterisk, posterior leaflet). F, Histologic evaluation showing focal myxoid degeneration, with fragments of partially hyalinized fibrous connective tissue and extensive amorphous dystrophic calcification accompanied by chronic inflammation, including histiocytes, macrophages, neutrophils, and plasma cells.

culture-negative cases. ${ }^{4}$ Thus, a molecular method for the identification of Twhipplei is an important tool for the management of the uncommon forms of infective endocarditis. Immunohistochemical analysis for $T$ whipplei remains the reference standard for the diagnosis of Whipple's disease. It is a sensitive and specific method that can be easily performed in most laboratories. ${ }^{5}$ This method offers added specificity compared with polymerase chain reaction methods owing to the direct visualization of bacilli and antigens within the cells in the tissue sections. It also offers increased sensitivity and specificity compared with the traditional periodic acid-Schiff staining method. However, the systematic use of polymerase chain reaction amplification of the bacterial 16 rRNA gene and subsequent sequencing in valvular tissue has been increasing the percentage of patients diagnosed with Twhipplei endocarditis. ${ }^{4}$ The correct diagnosis of $T$ whipplei infection is critical, because it will guide postoperative antimicrobial treatment in the prevention of recurrent endocarditis. The latter occurs commonly without prolonged antibiotic treatment and then 
will be hard to eradicate, and reoperations have resulted in substantial morbidity and mortality.

\section{CONCLUSIONS}

The polymerase chain reaction method adds substantially to the etiologic diagnosis of infective endocarditis, leading to institution of the appropriate antibiotic therapy. It should be considered for all patients for whom routine cultures have been negative despite a high index of clinical suspicion of infective endocarditis.

\section{References}

1. Fenollar F, Puéchal X, Raoult D. Whipple's disease. N Engl J Med. 2007;356:55-66

2. Fenollar F, Célard M, Lagier JC, Lepidi H, Fournier PE, Raoult D. Tropheryma whipplei endocarditis. Emerg Infect Dis. 2013;19:1721-30.

3. Amendolara M, Barbarino C, Bucca D, Stevanato G, Zucchelli M, Romano F, et al. Whipple's disease infection surgical treatment: presentation of a rare case and literature review. G Chir. 2013:34:117-21.

4. Geissdörfer W, Moos V, Moter A, Loddenkemper C, Jansen A, Tandler R, et al High frequency of Tropheryma whippley in culture negative endocarditis. J Clin Microbiol. 2012;50:216-22.

5. Baisden BL, Lepidi H, Raoult D, Argani P, Yardley JH, Dumler JS. Diagnosis of Whipple disease by immunohistochemical analysis: a sensitive and specific method for the detection of Tropheryma whipplei (the Whipple bacillus) in paraffin-embedded tissue. Am J Clin Pathol. 2002;118:742-8.

\title{
Successful aortic valve repair after trauma-induced aortic regurgitation
}

\author{
Hassane Abdallah, MD, Alaae Boutayeb, MD, and Gebrine El Khoury, PhD, Brussels, Belgium
}

Posttraumatic aortic valve regurgitation is uncommon, and aortic valve replacement has long been the sole therapeutic solution. Current data, however, suggest that aortic valve repair techniques represent a valuable option, particularly for young patients with favorable anatomic presentation. This article reviews posttraumatic aortic regurgitation and the importance of aortic valve repair techniques as a feasible and effective therapeutic option.

\section{CLINICAL SUMMARY}

We report the case of a 34-year-old man who was injured in a traffic accident. Results of the first evaluation after the accident were normal; however, the patient was seen 3 months later with progressive dyspnea. On physical examination, a new aortic diastolic murmur in the aortic area was heard, with no signs of heart failure. Echocardiographic findings (transthoracic and transesophageal echocardiography) showed an aortic valve prolapse associated with moderate aortic regurgitation (Figure 1, A). The prolapse was caused by a rupture of the right noncoronary commissure. This corresponds to type II regurgitation according to the classification of Boodhwani and associates. ${ }^{1}$

Because of the observed injuries and the young age of the patient, aortic valve repair was proposed. The procedure

\footnotetext{
From the Cardiothoracic and Vascular Surgery Department, Cliniques Universitaires Saint-Luc, Brussels, Belgium.

Disclosures: Authors have nothing to disclose with regard to commercial support.

Received for publication April 8, 2014; revisions received May 18, 2014; accepted for publication May 27, 2014; available ahead of print Aug 12, 2014.

Address for reprints: Hassane Abdallah, MD, 10500 Boulevard de l'Acadie,

820 H4N2V4 Montreal, Canada (E-mail: abdallahhassane@hotmail.com).

J Thorac Cardiovasc Surg 2014;148:e197-8

$0022-5223 / \$ 36.00$

Copyright (c) 2014 by The American Association for Thoracic Surgery

http://dx.doi.org/10.1016/j.jtcvs.2014.05.086
}

was carried out through a median sternotomy and under normothermic cardiopulmonary bypass. After aortic crossclamping, the aorta was opened and the valve inspected. Operative findings confirmed the echocardiographic data (Figure 2, A). The aortic valve repair consisted of a reinsertion of the right noncoronary commissure with a 5-0 Prolene suture (Ethicon, Inc, Somerville, NJ) reinforced with autologous pericardium. In addition to noncoronary cusp shaving, a sub-commissural annuloplasty was also performed with pledgeted $2-0$ braided sutures. The first arm of the suture was passed from the aortic to the ventricular side, in the interleaflet triangle, and it came back out to the aortic side at the same level. Similarly, the second arm was passed just below the first. This annular reduction was calibrated with a 25-mm Hegar dilator and involved all the interleaflet triangles (Figure 2,B).

Postoperatively, all echocardiographic controls showed a good result with no residual regurgitation (Figure 1, $B$ ). The subsequent course was uneventful, and the patient was discharged on the sixth postoperative day with a regimen of aspirin only. Antiplatelet therapy was interrupted after 3 months. At 1-year follow-up, the patient was doing well with trivial aortic regurgitation.

\section{DISCUSSION}

Posttraumatic aortic regurgitation is a rare condition. A report of 546 patients who died after severe blunt chest trauma showed that only $0.73 \%$ of them had an injury to the aortic valve. ${ }^{2}$ These lesions are due mainly to a brutal increase of the intrathoracic pressure during early diastole, when the transaortic gradient is maximal. According to the available data, the noncoronary cusp seems to be the most frequently involved cusp. ${ }^{2,3}$ 